\title{
Assessment and Management of Right Ventricular Failure in Left Ventricular Assist Device Patients
}

\author{
William L. Holman, MD; Deepak Acharya, MD; \\ Franjo Siric, MD; Renzo Y. Loyaga-Rendon, MD, PhD
}

\begin{abstract}
Mechanical circulatory support devices, including ventricular assist devices (VADs) and the total artificial heart, have evolved to become accepted therapeutic options for patients with severe congestive heart failure. Continuous-flow left VADs are the most prevalent option for mechanical circulatory assistance and reliably provide years of support. However, problems related to acute and chronic right heart failure in patients with left VADs continue to cause important mortality and morbidity. This review discusses the assessment and management of right ventricular failure in left VAD patients. The goal is to summarize current knowledge and suggest new approaches to managing this problem. (Circ J 2015; 79: 478-486)
\end{abstract}

Key Words: Congestive heart failure; Right ventricle; Ventricular assist devices

$\mathbf{M}$ echanical circulatory support devices (MCSD), including ventricular assist devices (VADs) and the total artificial heart (TAH), have evolved to become accepted therapeutic options for patients with severe functional limitations because of congestive heart failure (Figure 1). Common indications for MCSD are bridge to transplantation (BTT), bridge to candidacy and destination therapy (DT) (Table). This review discusses the preoperative assessment and perioperative management of right ventricular (RV) failure in patients with a left VAD (LVAD).

Topics relevant to mechanical circulatory support (MCS) can be studied most effectively by analysis of prospectively gathered data from multiple institutions. The Interagency Registry for Mechanically Assisted Circulatory Support (INTERMACS) is an example of a prospective multicenter database. INTERMACS has a sufficiently large sample of patients with MCSDs to draw helpful inferences regarding their usage and answer important questions regarding patient outcomes (eg, determine risk factors for morbidity and mortality). Another important role for INTERMACS is to establish definitions for adverse events that may occur in patients with MCSDs. ${ }^{1}$

The present review will use the INTERMACS definition for RV failure (Appendix S1). The diagnosis of RV failure requires the presence of elevated central venous pressure (CVP), and clinical or laboratory manifestations of elevated CVP. INTERMACS lists 4 levels of RV failure (mild, moderate, severe and severe-acute). The basis for the severe and severeacute diagnoses of RV failure is a need for mechanical RV assistance at any time during support, or death related to RV failure.
RV failure and a requirement for biventricular (bi-VAD) support have long been recognized as risk factors for death in VAD recipients. ${ }^{2-4}$ The $6^{\text {th }}$ Annual INTERMACS Report ${ }^{5}$ analyzed early and late risk factors for death in 9,372 patients. The following risk factors for early death were found: higher right atrial pressure (ie, CVP) at the time of implant (hazard ratio [HR], 1.11); RVAD placement at the same operation as LVAD placement (HR, 2.45); higher serum bilirubin prior to surgery (HR, 1.21); and the presence of ascites prior to surgery (HR, 1.27).

The adverse influence of bi-VAD support as compared with LVAD support is evident in pulsatile flow (PF) as well as continuous-flow (CF) VADs (Figures 2,3). ${ }^{5}$ Data from 10,542 MCSD implants spanning June 2006 through December 2013 showed 1-year post-implant survival of $81 \%$ for CF LVADs, but only $57 \%$ survival for CF bi-VAD patients. These survival figures represent an improvement from earlier results; however, the adverse influence of RV mechanical assistance is still obvious.

Improvements in survival from the PF pump era to the era of CF pumps reflect advances in pump design, refinement of implantation techniques and the management of MCSD patients; yet there is still a $20 \%$ worse 1-year survival in patients who require mechanical RV support in addition to a LVAD. The adverse effect of RV failure that is sufficiently severe to require MCS has not improved in the era of $\mathrm{CF}$ assist devices. It is striking that from the years 2008 to 2010 until the years 2011 to 2013, there actually was a worsening of survival in patients with a CF LVAD who required mechanical RV support (Figure 3). ${ }^{5}$ When the survival for patients with CF VADs is stratified according to the severity of $\mathrm{RV}$ dysfunction, a

Received January 29, 2015; accepted January 29, 2015; released online February 17, 2015

Division of Cardiothoracic Surgery (W.L.H., F.S.), Division of Cardiovascular Disease (D.A., R.Y.L.-R.), University of Alabama at Birmingham, Birmingham, AL, USA

Mailing address: William L. Holman, MD, Division of Cardiothoracic Surgery, University of Alabama at Birmingham, $70319^{\text {th }}$ Street South, Room 719, Birmingham, AL 35294, USA. E-mail: wholman@uab.edu

ISSN-1346-9843 doi:10.1253/circj.CJ-15-0093

All rights are reserved to the Japanese Circulation Society. For permissions, please e-mail: cj@j-circ.or.jp 


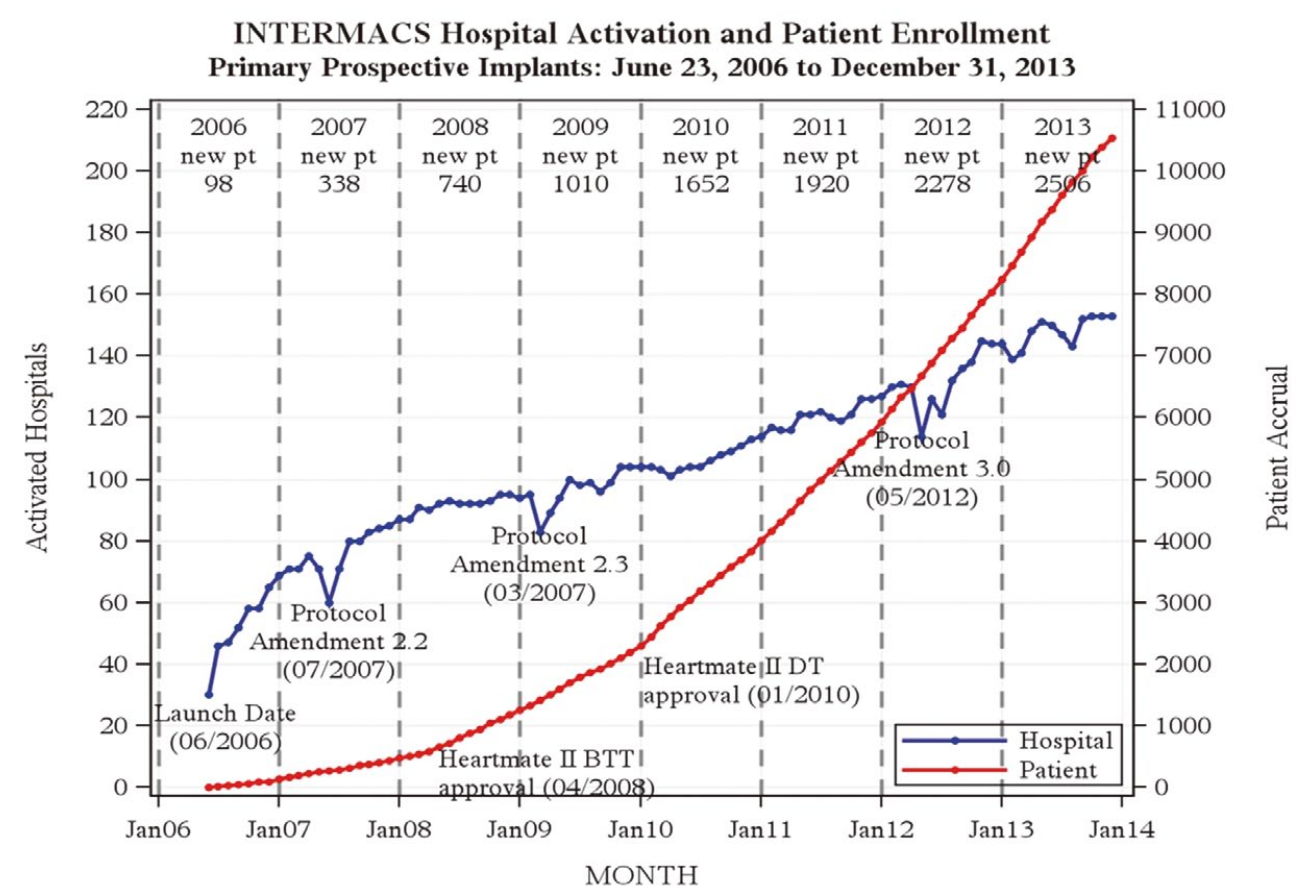

Figure 1. Growth in numbers of implantation centers and numbers of patients (pt) receiving mechanical circulatory support devices from June 23, 2006 through December 31, 2013 according to data from the Interagency Registry for Mechanically Assisted Circulatory Support (INTERMACS). Reproduced with permission from Kirklin JK, et al. ${ }^{5}$

\begin{tabular}{|c|c|c|c|c|c|c|c|c|}
\hline \multirow{4}{*}{$\begin{array}{l}\text { Device strategy at time } \\
\text { of implantation }\end{array}$} & \multicolumn{8}{|c|}{ June 2006-December 2013 ( $n=10,542)$} \\
\hline & \multicolumn{6}{|c|}{ Implant era } & \multirow{2}{*}{\multicolumn{2}{|c|}{ Total }} \\
\hline & \multicolumn{2}{|c|}{ 2006-2007 } & \multicolumn{2}{|c|}{ 2008-2010 } & \multicolumn{2}{|c|}{ 2011-2013 } & & \\
\hline & $\mathbf{n}$ & $\%$ & $\mathbf{n}$ & $\%$ & $\mathbf{n}$ & $\%$ & $\mathbf{n}$ & $\%$ \\
\hline BTT & 185 & 42.4 & 1,335 & 39.2 & 1,453 & 21.7 & 2,973 & 28.2 \\
\hline BTC & 162 & 37.1 & 1,325 & 39 & 2,373 & 35.4 & 3,860 & 36.7 \\
\hline $\mathrm{DT}^{*}$ & 64 & 14.7 & 666 & 19.6 & 2,786 & 41.6 & 3,516 & 33.4 \\
\hline BTR & 17 & 3.9 & 38 & 1.1 & 38 & 1.0 & 93 & 0.9 \\
\hline Rescue therapy & 8 & 1.8 & 24 & 1.0 & 28 & 0.4 & 60 & 0.6 \\
\hline Other & 0 & 0.0 & 14 & 0.4 & 26 & 0.4 & 40 & 0.4 \\
\hline Total & 436 & 100.0 & 3,402 & 100.0 & 6,704 & 100.0 & 10,542 & 100.0 \\
\hline
\end{tabular}

*Increase in destination therapy from 2006 to 2013. BTC, bridge to candidacy; BTR, bridge to recovery; BTT, bridge to transplantation; DT, destination therapy; INTERMACS, Interagency Registry for Mechanically Assisted Circulatory Support. Reproduced with permission from Kirklin JK, et al. ${ }^{5}$

statistically significant adverse effect of RV failure is evident (Figure 4). ${ }^{5}$ One can rationally argue that RV failure is one of the most important problems currently facing the field of MCS.

\section{Preoperative Assessment of Patients Receiving VADs: Emphasis on the RV}

Analysis of patient outcomes from INTERMACS has defined the risk factors for death based on multivariate analysis. ${ }^{6}$ The risk factors included the presence of ascites prior to implantation of a MCSD (relative risk [RR], 2.04; $\mathrm{P}=0.003$ ), higher serum bilirubin prior to implantation $(\mathrm{RR}, 1.49 ; \mathrm{P}=0.05)$ and the use of biventricular assistance (RR, 2.12; P=0.002). Similar risk factors have been noted in other retrospective multivariate analyses of death in recipients of MCSDs. This reflects the important adverse effects of RV failure on survival, including impaired hepatic function as indicated by elevated bilirubin, presence of ascites, hypoalbuminemia, or elevated international normalized ratio (INR). Furthermore, the importance of RV failure to the survival of patients with MCSDs suggests that establishing LVAD support relatively early after the onset of advanced heart failure (ie, NYHA Class IV functional status) will improve survival by avoiding the sequelae 


\section{Intermecs Implants: June 2006 - December 2013, n=10542}

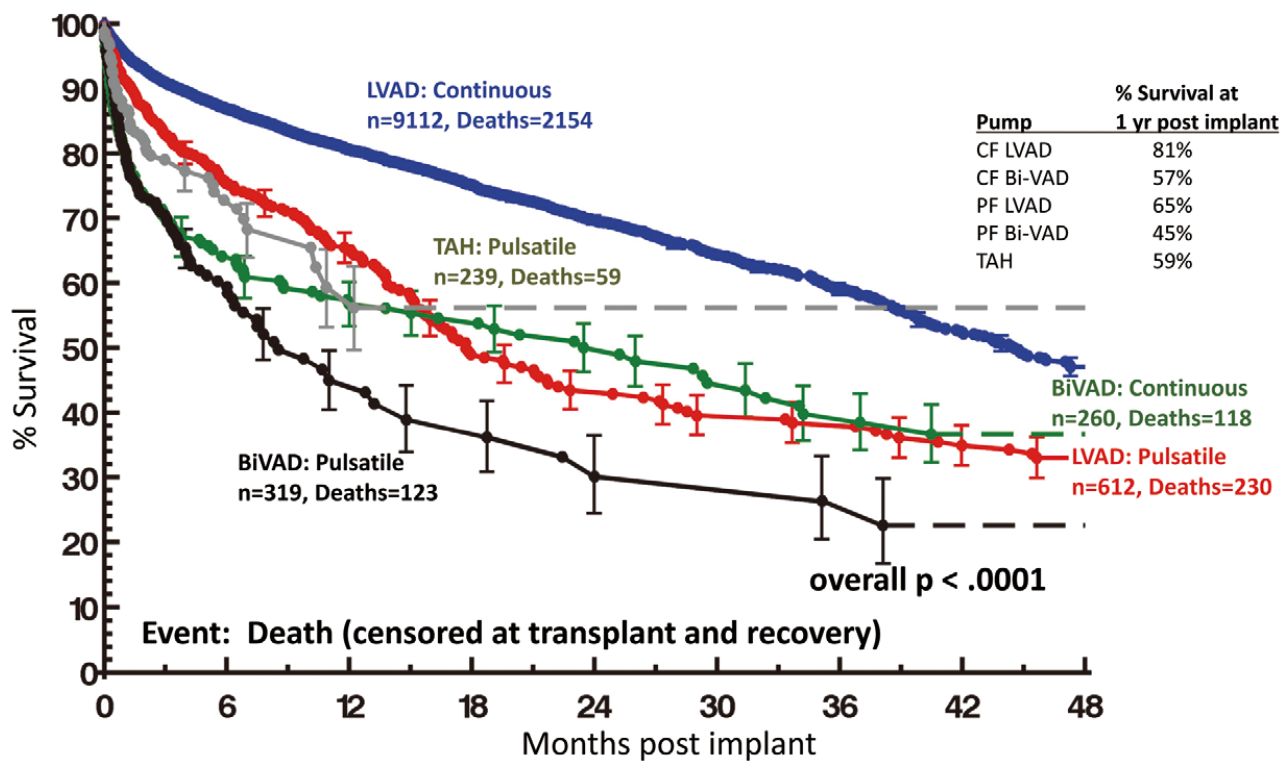

Figure 2. Survival of patients with CF or pulsatile bi-VADs is less than those with LVAD only according to data from the Interagency Registry for Mechanically Assisted Circulatory Support (INTERMACS). CF, continuous flow; LVAD, left ventricular assist device; PF, pulsatile flow; TAH, total artificial heart. Reproduced with permission from Kirklin JK, et al. ${ }^{5}$

\section{Intermecs Continuous Flow LVAD/BiVAD Implants: 2008 - 2013, n=9372}

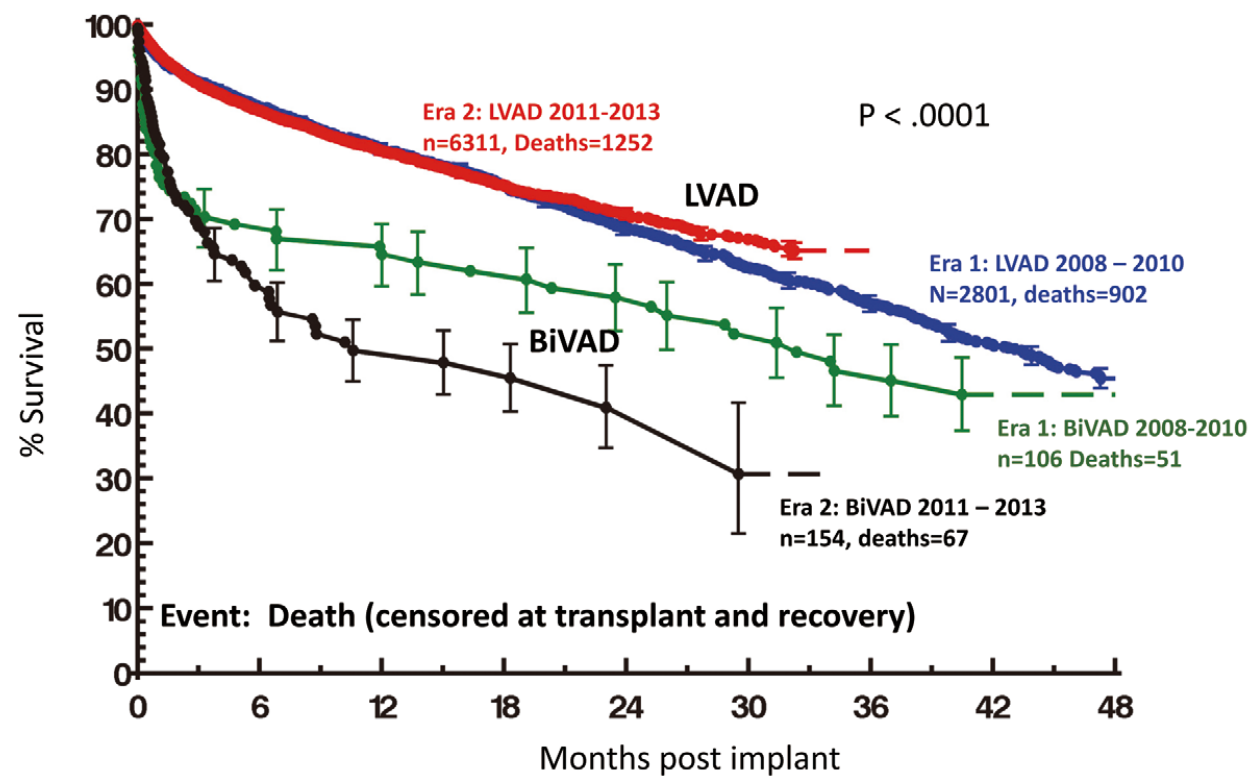

Figure 3. Comparison of 2 eras shows that survival of bi-VAD patients has worsened in the most recent era, and that LVAD patient survival continues to be superior to bi-VAD patient survival, according to data from the Interagency Registry for Mechanically Assisted Circulatory Support (INTERMACS). LVAD, left ventricular assist device. Reproduced with permission from Kirklin JK, et al. ${ }^{5}$ 


\section{Intermecs Continuous Flow LVAD/BiVAD Implants: 2008 - 2013, n=9372}

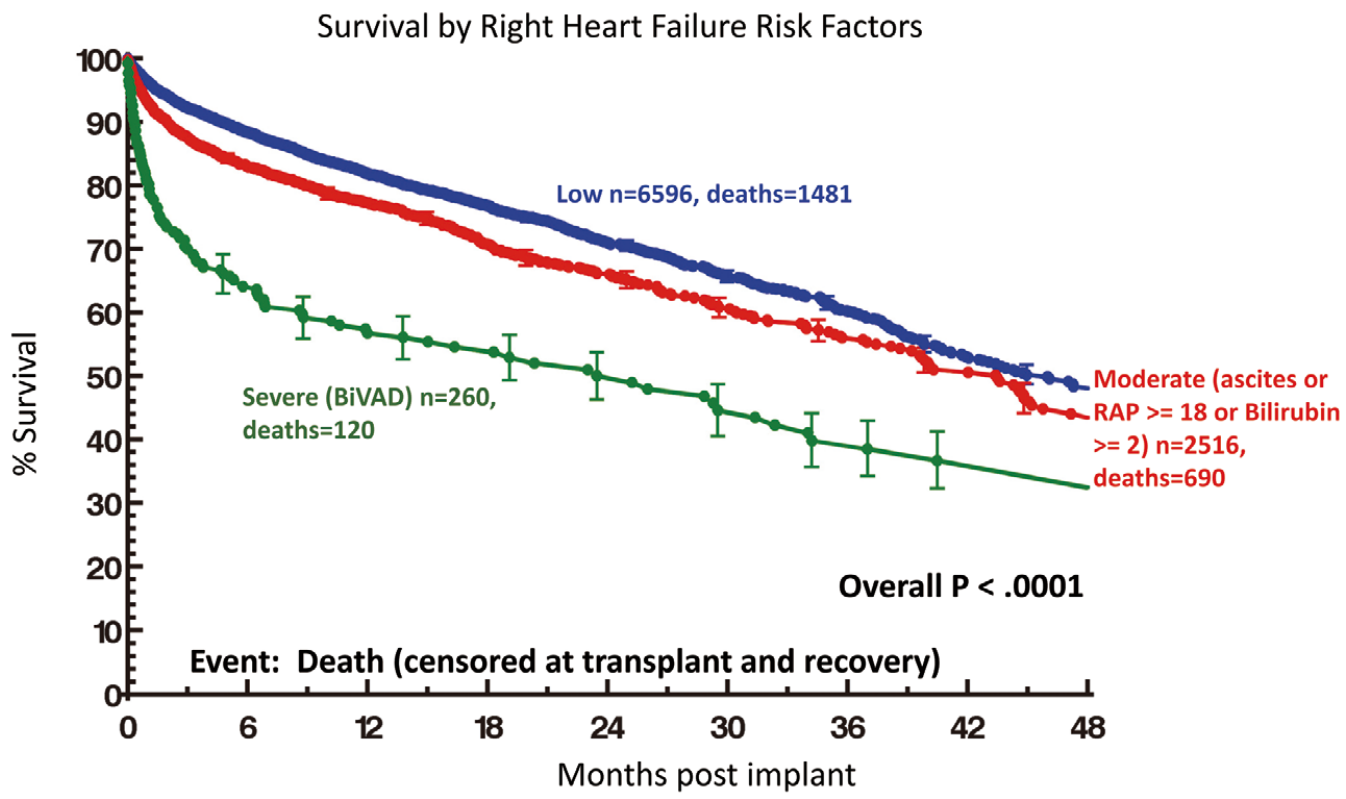

Figure 4. In the patient cohort implanted from 2008 to 2013 (continuous-flow LVAD and bi-VAD patients) severity of right heart failure as determined by mean right atrial pressure (RAP) $\geq 18 \mathrm{mmHg}$, serum bilirubin $\geq 2 \mathrm{mg} \%$, presence of ascites on physical examination, or use of a RVAD (ie, bi-VAD patient) were risk factors for death, according to data from the Interagency Registry for Mechanically Assisted Circulatory Support (INTERMACS). LVAD, left ventricular assist device; RAP, right atrial pressure Reproduced with permission from Kirklin JK, et al. ${ }^{5}$

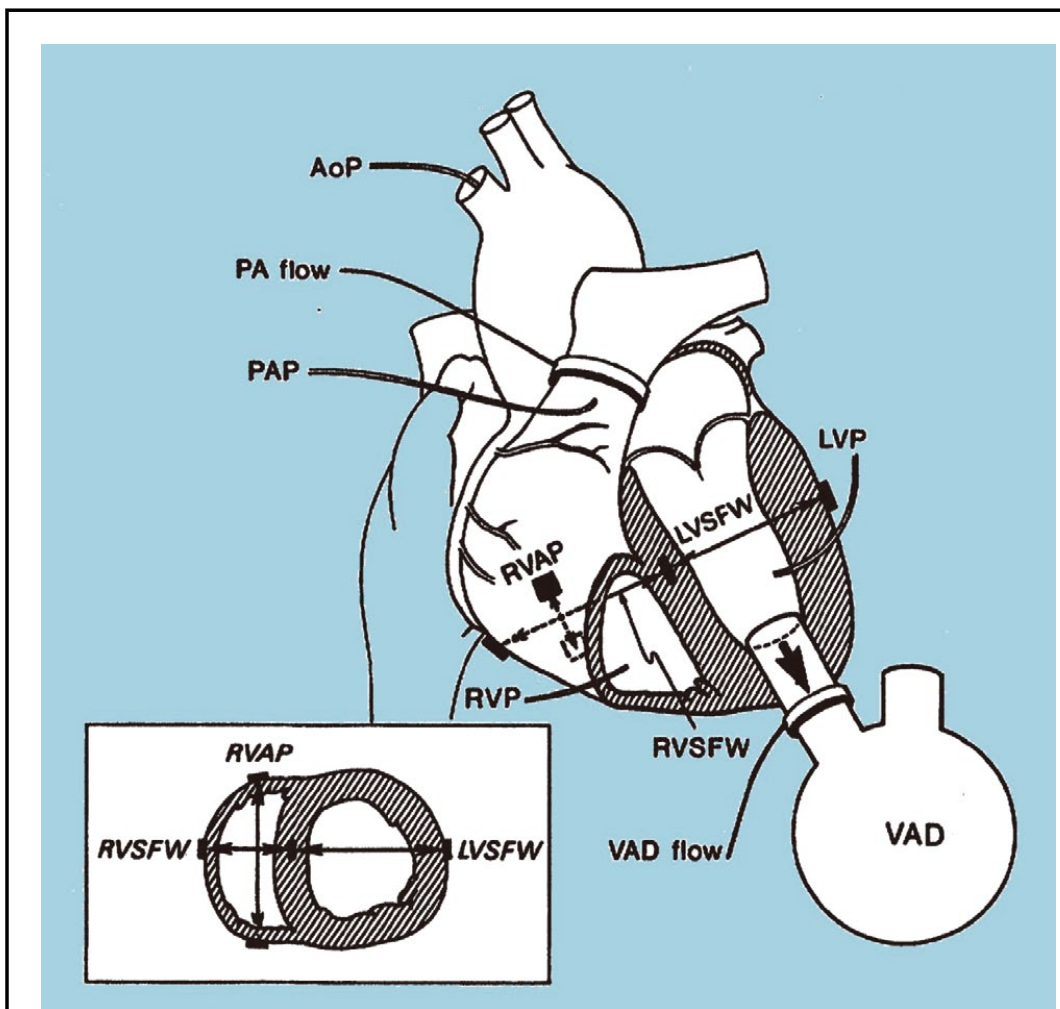

Figure 5. In a study by Woodard et al, the effect of left ventricular (LV) systolic unloading on right ventricular (RV) function was measured. AoP, aortic pressure; PAP, pulmonary artery pressure; PA, pulmonary artery; LVP/RVP, LV/RV pressures as measured with pressure-tip transducers; sonomicrometry pairings for dimensions: RVAP, RV anterior-posterior; L/RVSFW, left/right ventricle septum-free wall; VAD, ventricular assist device. Reproduced with permission from Woodward JC, et al. ${ }^{15}$ 
of RV failure and the need for a RVAD. ${ }^{6}$

Scoring systems and risk factors for predicting RV failure and patient survival following LVAD placement have been published. In one such scoring system, Cowger and associates 7 developed a model to predict survival in patients that received HeartMate II LVADs (Thoratec, Pleasanton, CA, USA). Their study included patients enrolled in the FDA trials of the HeartMate II LVAD for BTT or DT. The 1,122 patients included in those trials were randomly divided into a cohort for derivation of the HeartMate II Risk Score (HMRS) designed to predict 90-day mortality, and another cohort to validate the predictive model. The multivariable predictors of 90-day mortality for the HMRS included age; serum albumin level; serum creatinine level; INR; and LVAD implantation at less experienced centers. Three of these risk factors (hypoalbuminemia, serum creatinine and coagulopathy reflected by an increased INR) are associated with RV failure. ${ }^{7}$ In addition to developing the HMRS, the Cowger study compared the MHRS with other predictive models, including one based on the Model for End Stage Liver Disease (MELD) score. The HMRS only showed statistically significant superiority in the cohort of patients used to derive the HMRS and the entire sample of HeartMate II patients. This finding supports the notion that liver dysfunction typically associated with advanced RV and left ventricular (LV) failure comprises a substantial portion of the risk for death following placement of a LVAD.

There are retrospective single institution ${ }^{3,8,9}$ and multi-institution $^{2,10,11}$ analyses of risk factors for RV failure following placement of a LVAD. A common theme of the studies is that clinical indicators of RV failure (eg, ascites) and consequent hepatic insufficiency (eg, elevated bilirubin or INR) are associated with increased morbidity and mortality in patients managed with VADs.

\section{RV and LV Interactions: Understanding the Effects of LVAD Support on RV Function}

The physiology of RV contractile function in the setting of advanced heart disease is complex ${ }^{12}$ because it is influenced by several factors, including LV end-diastolic pressure; pulmonary vascular resistance (PVR) and pulmonary artery pressure (PAP); coronary artery perfusion; and mechanical interactions of the right and left ventricles. The following comments focus on the septal contribution to RV function. Understanding the mechanical coupling between the right and left ventricles is important for optimizing LVAD pumping (ie, setting the revolutions per minute [rpm]) to avoid acute RV failure at the time of separating from cardiopulmonary bypass (CPB) and during the immediate postoperative period.

Early studies predicted that LVAD pumping would improve the diastolic function of the RV and this improvement would nullify the loss of septal contractile force that otherwise contributes to RV systolic function. ${ }^{13}$ A pair of elegant studies in porcine hearts modified this notion (Figure 5). ${ }^{14,15}$ In summary, these experiments showed that (1) in the failing as opposed to the normal heart, losing the septal contribution to systolic RV function outweighs any benefit from improved RV diastolic function ${ }^{14}$ and (2) even in the normal heart, a small but measurable decrease in RV systolic function occurs with LV unloading. ${ }^{15}$

The overall importance of the septum to global RV function remains controversial because there is evidence for improved RV function following initiation of LVAD support. ${ }^{16,17}$ The issue is further complicated by the beneficial effects of medications such as nitric oxide ${ }^{18}$ or phosphodiesterase- 5 inhibitors ${ }^{19}$ on RV afterload, and improvements in PVR that occur in response to chronic lowering of $\mathrm{LV}$ end-diastolic pressure because of a LVAD. However, there is now general agreement that setting the speed (ie, rpm) of a rotary pump too high will cause displacement of the ventricular septum towards the LV, and this displacement will adversely affect RV contractile function by decreasing the septal contribution to RV contractility.

\section{Pre- and Perioperative Assessment of RV Function: History, Physical Examination, Hemodynamic Measurements, and Echocardiography}

Patients should be asked specific questions regarding the acuity and severity of their heart failure, and whether they have experienced lower extremity edema, abdominal swelling, or jaundice. Clues to important RV failure on examination include the severity and extent of lower extremity edema; ascites; hepatomegaly; pulsatile liver; and scleral icterus. Signs of chronic and severe RV failure indicate a higher perioperative risk for MCS.

The laboratory and imaging studies to evaluate a patient prior to support with a LVAD include right and left heart catheterization, cardiac echocardiography, and blood tests. Blood tests include studies to determine nutritional status (serum protein, albumin and pre-albumin levels), renal function (serum blood urea nitrogen and creatinine levels) and hepatic function (prothrombin time/INR, levels of total and fractionated bilirubin, serum alkaline phosphatase, alanine aminotransferase and aspartate aminotransferase). Nutritional deficiencies occur in patients with severe heart failure independently of severe RV failure; however, RV failure may substantially contribute to nutritional deficiencies via hepatic dysfunction secondary to passive hepatic congestion or protein-losing enteropathy.

Cardiac catheterization includes determination of pulmonary capillary wedge pressure (PCWP), right atrial pressure (RAP), PAP and Fick cardiac output. RAP $>20 \mathrm{mmHg}$ and a high RAP relative to the PCWP are clues regarding the severity of RV impairment. Preimplant management can often lower the RAP to an acceptable range of $\leq 15 \mathrm{mmHg}$. Such a response suggests that RV function will be adequate to support a LVAD; ${ }^{20}$ however, research is still needed to fully quantify this risk reduction. The RV stroke work index (RVSWI) is a measurement of RV function RVSWI=(meanPAP-meanRAP $) *(\mathrm{SVI})$. A low RVSWI has been associated with increased risk for severe RV failure requiring RVAD implantation. ${ }^{8}$

Echocardiographic evaluation of RV function is challenging and requires integration of multiple parameters and techniques. ${ }^{21}$ The challenge is partly related to acute and chronic changes in RV geometry. Normally, the RV has a crescentlike morphology, but in the setting of heart failure, the RV becomes more spherical. In the case of acute RV failure associated with LVAD pumping, shifting of the ventricular septum because of LV unloading further complicates measurement of RV function. These challenges have been recognized and described by others. ${ }^{22}$

Echocardiographic evaluation of RV morphology and contractile function begins with accurate measurement of the ventricular dimensions. Measures of RV mechanical performance must take into account the presence and severity of tricuspid and pulmonary valve pathologies in addition to PAP (ie, RV afterload). Systolic function is often measured solely as the RV ejection fraction, which is sensitive to RV afterload, preload, valvular dysfunction, and inotropic stimulation. 
Other metrics for RV function are derived from RV (ie, tricuspid) annular motion over time. These include the tricuspid lateral annular systolic velocity ( $\left.S^{\prime}\right), R V$ index of myocardial performance, 2D RV fractional area change and tricuspid annular plane systolic excursion (TAPSE). ${ }^{21}$ Using another approach, Topilsky et al demonstrated that early systolic equalization of the RV and RA pressures is associated with increased mortality and morbidity after LVAD implantation. ${ }^{23}$

TAPSE is measured from the 4-chamber view of the heart, and higher TAPSE values are associated with better RV contractile function. TAPSE is relatively simple and reproducible as compared with other metrics (eg, speckle-tracking measures of longitudinal RV strain). The main disadvantage of TAPSE is shared with other regional measurements of ventricular function, namely regional measurements do not include information from unsampled regions of the heart that might importantly modify the calculated measurement.

Speckle-tracking echocardiography increases the precision of measuring RV contractile function. ${ }^{24-26}$ Speckle-tracking measurements of RV function and improvements in preimplant RV function after intensive medical therapy may be especially useful for predicting RV failure following LVAD implantation. ${ }^{17,20,25}$ In fact, recent studies have shown that the global longitudinal strain of the RV free wall represents a new parameter that may help predict the occurrence of RV failure post LVAD. Unfortunately, the time required for analysis of speckle-tracking data limits its usefulness for intraoperative measurements. This may change in the future with automation for speckle-tracking measurements.

There is general agreement that intraoperative transesophageal echocardiography (TEE) should be routinely used during the implantation of LVADs, ${ }^{27}$ unless there is a contraindication to the use of TEE (eg, esophageal stricture). Using TEE to measure RV geometry, tricuspid incompetence, and RV contractile function can help determine the risk for perioperative RV failure at the time of LVAD implantation. ${ }^{20,25,28-31}$ Decisions regarding pharmacological management of RV function and PVR, as well as decisions regarding initiation of MCS for the RV cannot be accurately predicted based on echocardiographic information alone. Moreover, there is no single algorithm that accurately defines optimal management of all MCSD patients in detail. Iterative assessment and decision making during and after the implantation of a LVAD is the most effective method of optimizing RV function. Iterative assessments are also likely to provide the best timing for placement of a RVAD should one be necessary, rather than depending on a decision made prior to starting the LVAD implantation operation. Assessment of the inflow cannula position and flow into the cannula are important for optimizing RV function by ensuring optimal decompression of the LV.

\section{Management of RV Failure: Preoperative Measures}

Patients with chronic heart failure may have acute exacerbations related to progression of cardiac disease, a new myocardial infarction, myocardial ischemia, or sepsis. If the patient has life-threatening hemodynamic instability, the most expeditious course of management is emergency initiation of extracorporeal membrane oxygenation (ECMO) support.

After a patient is stabilized on ECMO, intensive management of heart failure with inotropes and diuretics is useful for improving for RV function prior to LVAD placement. Several analyses have shown that INTERMACS level 1 status is a risk factor for death following LVAD placement. At the present time, there are no data to prove that conversion from INTERMACS level 1 to a less acutely ill profile (eg, 2 or 3 ) improves survival following LVAD placement. However, it is reasonable to assume that this is the case and avoid implantation of a durable LVAD in an acutely unstable level 1 patient.

For patients with exacerbation of existing heart failure but not requiring ECMO support, intensive medical therapy with inotropes, afterload reduction and diuretics can often reduce RAP and PCWP. Counterpulsation with an intraaortic balloon pump is another useful adjunctive measure. Diminishing acute distention of the RA and RV, together with improvement of end-organ dysfunction increases the chances for survival following LVAD placement.

\section{Management of RV Failure: Intraoperative Measures}

Avoidance of air embolism to the right coronary artery and preventing leftward shifting of the ventricular septum from over-pumping by the LVAD are important measures to avoid iatrogenic injury to the RV during LVAD placement.

It is important to understand the acute effects of LVAD pumping on valvular function. Aortic valve insufficiency becomes more severe as the LV pressures are diminished by LVAD pumping. Mitral insufficiency typically decreases on initiation of LVAD support, whereas tricuspid insufficiency may increase as venous return to the RV increases. ${ }^{32} \mathrm{~A}$ mild to moderate degree of mitral insufficiency is usually well tolerated in patients with LVADs. The question of whether or not to repair or replace a moderately or severely regurgitant tricuspid valve is complex, with conflicting opinions. ${ }^{33-37}$ Unfortunately, no studies have taken into account RV contractility in the determination of whether tricuspid repair does or does not influence survival following LVAD placement. The absence of an easily obtained metric for RV contractility (ie, loadindependent RV systolic function) contributes to the confusion over the appropriateness of valve repair, because tricuspid valve competency becomes irrelevant if RV contractility is profoundly diminished. This is the case in patients with moderate to severe tricuspid insufficiency with mean RAPs that are nearly equivalent to the mean PAPs. On the other hand, patients with severe tricuspid insufficiency in the face of moderately or markedly elevated PAPs may respond well to tricuspid valve repair. Future research will clarify this point.

Most patients require infusion of inotropic agents, such as milrinone and epinephrine, to support RV function during the immediate perioperative period. Inhaled nitric oxide and epoprostenol that decrease PVR can further improve RV function by diminishing RV afterload and have been used in patients undergoing LVAD implantation..$^{38,39}$ Another important point is strict ventilator management. Maintaining mild respiratory alkalosis and adequate oxygenation encourages pulmonary vasodilation and therefore reduces RV afterload.

\section{RV Support: MCS Options}

Before choosing a MCSD for RV support, the surgeon must define the need. Specifically, what is the anticipated duration of RV mechanical support? Will support of pulmonary function (ie, gas exchange) be necessary? Is peripheral or central cannulation of the right-sided circulation best for the specific clinical situation? The answer to these questions will determine the best choice for each patient.

If the potential for pulmonary support is high, then a paracorporeal pump should be chosen so that a gas exchange 


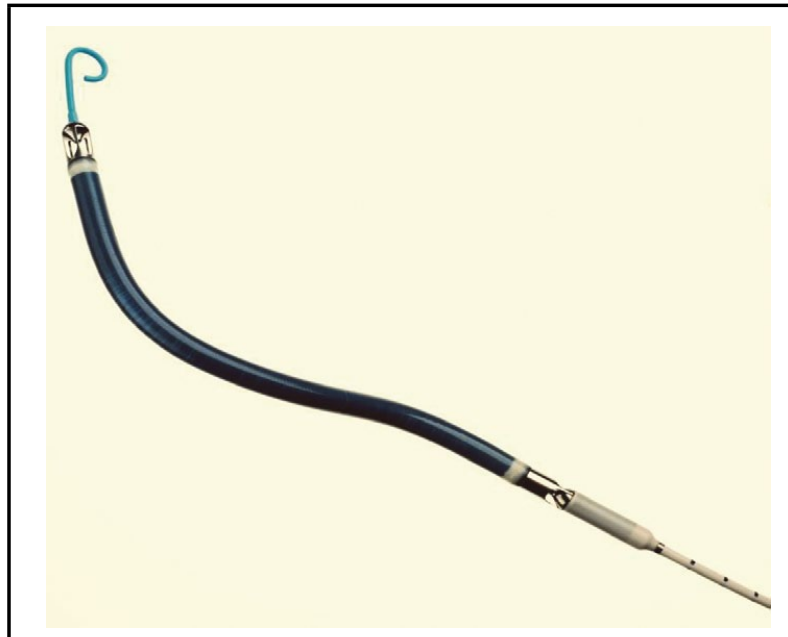

Figure 6. The Impella RP LVAD (Abiomed, Danvers, MA, USA) is passed from the right femoral vein antegrade across the tricuspid and pulmonary valves so that the pump pulls blood from the right atrium-inferior vena cava junction into the main pulmonary artery.

device can be spliced into the RVAD circuit. Centrifugal pumps (eg, Levitronix ${ }^{\mathrm{TM}}$, Thoratec, Pleasanton, CA, USA; or Rotaflow $^{\mathrm{TM}}$, MAQUET Cardiovascular LLC, San Jose, CA, USA) serve this purpose well. For a right paracorporeal VAD circuit, a venous return cannula can be placed percutaneously via the femoral vein into the RA or the cannula can be placed directly into the RA. RVAD outflow is achieved with an 8- or 10-mm Dacron graft sewn to the main pulmonary artery. Some surgeons favor the use of a percutaneous venous return line with a long 8- or 10-mm Dacron graft that exits the closed chest. This option allows removal of the RVAD cannulas at the bedside.

An intriguing new catheter-based rotary pump has completed initial trials and is awaiting approval by the FDA (Impella RP ${ }^{\mathrm{TM}}$ VAD, Abiomed, Danvers, MA, USA). This pump is similar to the Impella pumps that are used for LV assistance (Figure 6). The Impella RP VAD is deployed over a wire that guides the device through the tricuspid and pulmonary valves and into the main pulmonary artery. The motor and impeller are located at the tip of the device. Blood enters the pump at the RA-inferior vena cava junction and exits into the main pulmonary artery. If the user ensures correct positioning of the catheter and constant irrigation of the motor shaft, these devices reliably function for several days.

Paracorporeal pulsatile pumps (eg, Thoratec PVAD, Thoratec; and AB5000, Abiomed) can be used for longer term RV assistance (weeks to months). If the potential need is high for longterm RV mechanical support, one can simplify the implantation of a bi-VAD system by using the RVAD inflow cannula for venous return to the $\mathrm{CPB}$ circuit during LVAD implantation. For this operation, the LVAD is implanted and then an attempt is made to separate from bypass using the LVAD alone. If this is successful, the RVAD inflow cannula is removed. If a RVAD is necessary, bypass is resumed and the RVAD outflow cannula is anastomosed to the main pulmonary artery. A paracorporeal pump primed with sterile saline is then attached to the RVAD outflow cannula. The patient is taken off $\mathrm{CPB}$ and the RVAD inflow cannula is attached to the RVAD pump (Figure 7). The demands for patient care with a hybrid bi-

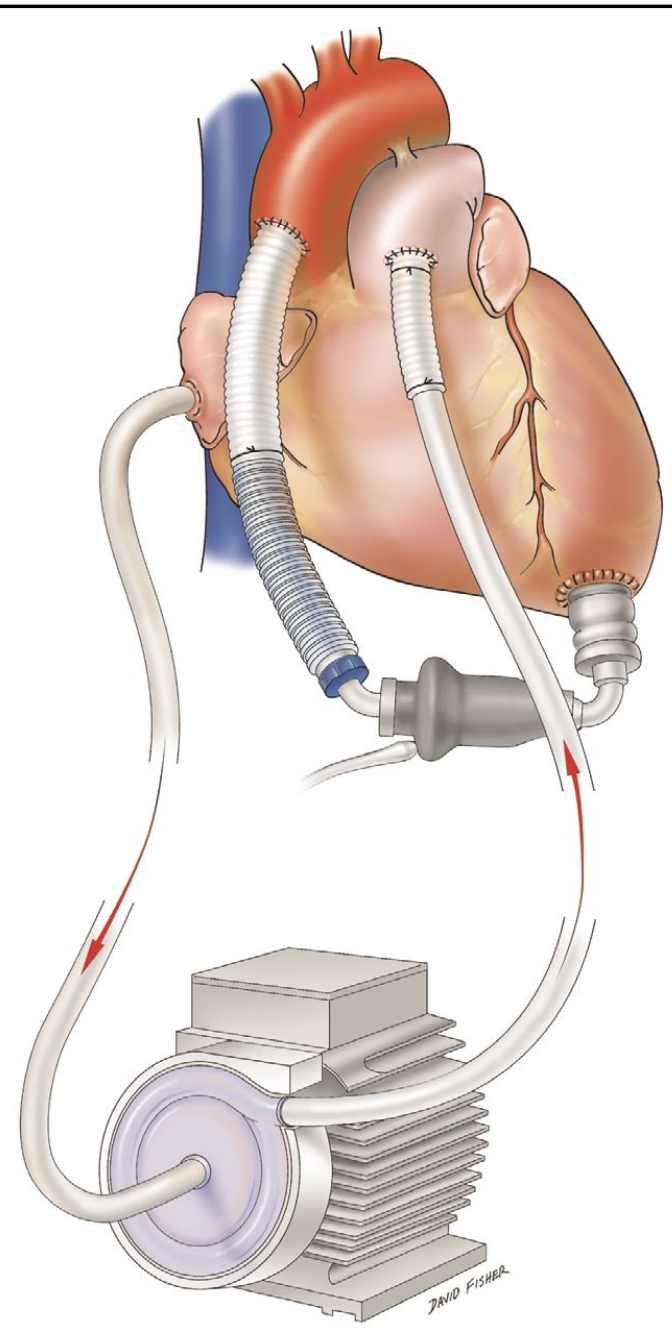

Figure 7. Hybrid bi-VAD system with a centrifugal flow paracorporeal right VAD with an axial flow intracorporeal left VAD. VAD, ventricular assist device.

VAD system (ie, a bi-VAD system with 2 types of pumps) are sufficiently challenging that living outside of the hospital is difficult.

Tector and associates described a novel method that uses an auxiliary CPB circuit to transition from full CPB to LVAD support ${ }^{40}$ The circuit draws blood from the RA and shunts it to the LV. The flow to the auxiliary circuit is balanced with the native circulation (ie, RV through lungs to $\mathrm{LV}$ ) to gradually wean the patient from the auxiliary CPB circuit. The slow weaning process provides oxygenated blood to the LVAD while partially off-loading the RV.

In another report, patients presenting with cardiogenic shock and placed emergently on ECMO with peripheral cannulation were managed with continued ECMO support following placement of a CF intracorporeal LVAD (average 4 days). ${ }^{41}$ The goal was to use partial ECMO support while the right heart and lungs recovered function.

Another solution to bi-VAD support involves the use of a HeartWare pump (HeartWare Inc, Framingham, MA, USA) as an implanted durable RVAD. ${ }^{42-46}$ Initially, surgeons and engineers felt that modification of the HeartWare outflow graft was necessary to mimic systemic vascular resistance. The 
modification was achieved by using sutures or surgical clips to decrease the diameter of the HeartWare outflow graft. The Berlin group used data from a mock circulation loop to determine that the optimal outflow graft diameter should be $5-7 \mathrm{~mm}$ over a length of $35 \mathrm{~mm}$ according to the patient's PVR. ${ }^{44}$ Notably, some surgeons consider that outflow graft modification is unnecessary.

The operative details for HeartWare RVAD inflow cannula placement vary. The Berlin group has placed the RVAD inflow cannula in the anterior free wall of the RV just below the RV outflow tract. Importantly, this group interposes 5-mm silicone-coated rings between the apical fixation ring and the surface of the RV. The silicone rings raise the sewing ring off the heart and shorten the length of the inflow cannula projecting into the RV. This maneuver diminishes the chance of suction against the opposing wall of the RV.

The diaphragmatic surface of the RV is another site chosen by some surgeons for placement of the HeartWare inflow cannula, while others have used RA cannulation. ${ }^{46}$ Another approach is to completely excise the heart at the level of the atrioventricular junction and implant 2 HeartWare pumps that are sutured to the tricuspid annulus or mitral annulus. In a single case report from Strueber and associates, a patient survived 14 weeks until cardiac transplantation with 2 HeartWare VADs that were implanted following removal of the patient's own heart. ${ }^{42}$

\section{Summary}

RV dysfunction has emerged as one of the most important problems facing patients with LVADs. Since its inception in 2006, analyses of INTERMACS data have allowed investigators to identify and quantify the risk factors for post-LVAD RV failure. However, methods for managing RV failure remain only partially successful.

There are 2 avenues for addressing the problems associated with RV failure in LVAD patients. The first is to develop implantable MCSDs designed specifically as RVADs. Ideally, these pumps will be small and have an inflow cannula that overcomes challenges related to the unique geometry of the $\mathrm{RV}$. A second avenue is to develop new medications or novel combinations of existing medications to acutely reduce $\mathrm{RV}$ afterload by reducing PVR. Elevated PVR related to chronic LV failure improves with mechanical support of the left-sided circulation, but this may take weeks or months.

Solving the problems associated with RV failure has the potential to substantially improve the survival and quality of life for patients with LVADs. The importance of this mission cannot be overstated, especially as the use of VADs as DT increases.

\section{References}

1. Arora D, Behr M, Pasquali M. A tensor-based measure for estimating blood damage. Artif Organs 2004; 28: $1002-1015$.

2. Farrar DJ, Hill JD, Pennington DG, McBride LR, Holman WL, Kormos RL, et al. Preoperative and postoperative comparison of patients with univentricular and biventricular support with the thoratec ventricular assist device as a bridge to cardiac transplantation. J Thorac Cardiovasc Surg 1997; 113: 202-209.

3. Fukamachi K, McCarthy PM, Smedira NG, Vargo RL, Starling RC, Young JB. Preoperative risk factors for right ventricular failure after implantable left ventricular assist device insertion. Ann Thorac Surg 1999; 68: 2181-2184.

4. Kavarana MN, Pessin-Minsley MS, Urtecho J, Catanese KA, Flannery M, Oz MC, et al. Right ventricular dysfunction and organ failure in left ventricular assist device recipients: A continuing problem. Ann Thorac Surg 2002; 73: 745-750.
5. Kirklin JK, Naftel DC, Pagani FD, Kormos RL, Stevenson LW, Blume ED, et al. Sixth INTERMACS Annual Report: A 10,000-patient database. J Heart Lung Transplant 2014; 33: 555-564.

6. Holman WL, Kormos RL, Naftel DC, Miller MA, Pagani FD, Blume E, et al. Predictors of death and transplant in patients with a mechanical circulatory support device: A multi-institutional study. J Heart Lung Transplant 2009; 28: 44-50.

7. Cowger J, Sundareswaran K, Rogers JG, Park SJ, Pagani FD, Bhat $\mathrm{G}$, et al. Predicting survival in patients receiving continuous flow left ventricular assist devices: The HeartMate II risk score. $J$ Am Coll Cardiol 2013; 61: 313-321.

8. Ochiai Y, McCarthy PM, Smedira NG, Banbury MK, Navia JL, Feng J, et al. Predictors of severe right ventricular failure after implantable left ventricular assist device insertion: Analysis of 245 patients. Circulation 2002; 106(Suppl 1): I198-I202.

9. Dang NC, Topkara VK, Mercando M, Kay J, Kruger KH, Aboodi MS, et al. Right heart failure after left ventricular assist device implantation in patients with chronic congestive heart failure. $J$ Heart Lung Transplant 2006; 25: 1-6.

10. Kormos RL, Teuteberg JJ, Pagani FD, Russell SD, John R, Miller LW, et al. Right ventricular failure in patients with the HeartMate II continuous-flow left ventricular assist device: Incidence, risk factors, and effect on outcomes. J Thorac Cardiovasc Surg 2010; 139: $1316-1324$

11. Drakos SG, Janicki L, Horne BD, Kfoury AG, Reid BB, Clayson S, et al. Risk factors predictive of right ventricular failure after left ventricular assist device implantation. Am J Cardiol 2010; 105: $1030-1035$.

12. Schwartz K, Singh S, Dawson D, Frenneaux MP. Right ventricular function in left ventrciluar disease: Pathophysiology and implications. Heart, Lung Circ J 2013; 22: 507-511.

13. Woodard JC, Farrar DJ, Chow E, Santamore WP, Burkhoff D, Hill JD. Computer model of ventricular interaction during left ventricular circulatory support. ASAIO Trans $1989 ; 35$ : 439-441.

14. Chow E, Farrar DJ. Right heart function during prosthetic left ventricular assistance in a porcine model of congestive heart failure. $J$ Thorac Cardiovasc Surg 1992; 104: 569-578.

15. Woodward JC, Chow E, Farrar DJ. Isolated ventricular systolic interaction during transient reductions in left ventricular pressure. Circ Res 1992; 70: 944-951.

16. Atluri P, Fairman AS, MacArthur JW, Goldstone AB, Cohen JE, Howard JL, et al. Continuous flow left ventricular assist device implant significantly improves pulmonary hypertension, right ventricular contractility, and tricuspid valve competence. J Card Surg 2013; 28: 770-775.

17. Herod JW, Ambardekar AV. Right ventricular systolic and diastolic function as assessed by speckle-tracking echocardiography improve with prolonged isolated left ventricular assist device support. $J$ Card Fail 2014; 20: 498-505.

18. Kukucka M, Potapov E, Stepanenko A, Weller K, Mladenow A, Kuppe $\mathrm{H}$, et al. Acute impact of left ventricular unloading by left ventricular assist device on the right ventricle geometry and function: Effect of nitric oxide inhalation. J Thorac Cardiovasc Surg 2011; 141: $1009-1014$.

19. Hamdan R, Mansour H, Nassar P, Saab M. Prevention of right heart failure after left ventricular assist device implantation by phosphodiesterase 5 inhibitor. Artif Organs 2014; 38: 963-967.

20. Verhaert D, Mullens W, Borowski A, Popovic ZB, Curtin RJ, Thomas JD, et al. Right ventricular response to intensive medical therapy in advanced decompensated heart failure. Circ Heart Fail 2010; 3: 340-346.

21. Rudski LG, Lai WW, Afilalo J, Hua L, Handschumacher MD, Chandrasekaran K, et al. Guidelines for the echocardiographic assessment of the right heart in adults: A report from the American Society of Echocardiography. J Am Soc Echocardiogr 2010; 23: 685-713.

22. Oe M, Gorcsan J III, Mandarino WA, Kawai A, Griffith BP, Kormos RL. Automated echocardiographic measures of right ventricular area as an index of volume and end-systolic pressure-area relations to assess right ventricular function. Circulation 1995; 92: 1026-1033.

23. Topilsky Y, Oh JK, Shah DK, Boilson BA, Schirger JA, Kushwaha SS, et al. Echocardiographic predictors of adverse outcomes after continuous left ventricular assist device implantation. JACC Cardiovasc Imaging 2011; 4: 211-222.

24. Blessberger H, Binder T. Two dimensional speckle tracking echocardiography: Basic principles. Heart 2010; 96: 716-722.

25. Cameli M, Lisi M, Righini FM, Focardi M, Lunghetti S, Bernazzali $\mathrm{S}$, et al. Speckle tracking echocardiography as a new technique to evaluate right ventricular function in patients with left ventricular assist device therapy. J Heart Lung Transplant 2013; 32: 424-430.

26. Kato TS, Jiang J, Schulze PC, Jorde U, Uriel N, Kitada S, et al. Serial 
echocardiography using tissue Doppler and speckle tracking imaging to monitor right ventricular failure before and after left ventricular assist device surgery. JACC Heart Fail 2013; 1: 216-222.

27. Patangi SO, George A, Pauli H, O'Leary D, Roysam C, Butt T, et al. Management issues during HeartWare left ventricular assist device implantation and the role of transesophageal echocardiography. Ann Card Anaesth 2013; 16: 259-267.

28. Puwanant S, Hamilton KK, Klodell CT, Hill JA, Schofield RS, Cleeton TS, et al. Tricuspid annular motion as a predictor of severe right ventricular failure after left ventricular assist device implantation. J Heart Lung Transplant 2008; 27: 1102-1107.

29. Potapov EV, Stepanenko A, Dandel M, Kukucka M, Lehmkuhl HB, Weng Y, et al. Tricuspid incompetence and geometry of the right ventricle as predictors of right ventricular function after implantation of a left ventricular assist device. J Heart Lung Transplant 2008; 27: $1275-1281$

30. Kukucka M, Stepanenko A, Potapov E, Krabatsch T, Redlin M, Mladenow A, et al. Right-to-left ventricular end-diastolic diameter ratio and prediction of right ventricular failure with continuous-flow left ventricular assist devices. J Heart Lung Transplant 2011; 30: 64-69.

31. Ky B, French B, Levy WC, Sweitzer NK, Fang JC, Wu AH, et al. Multiple biomarkers for risk prediction in chronic heart failure. Circ Heart Fail 2012; 5: 183-190.

32. Holman WL, Bourge RC, Fan P, Kirklin JK, Pacifico AD, Nanda NC. Influence of left ventricular assist on valvular regurgitation. Circulation 1993; 88: II-309-II-318.

33. Potapov EV, Schweiger M, Stepanenko A, Dandel M, Kukucka M, Vierecke J, et al. Tricuspid valve repair in patients supported with left ventricular assist devices. ASAIO J 2011; 57: 363-367.

34. Robertson JO, Grau-Sepulveda MV, Okada S, O'Brien SM, Matthew $\mathrm{BJ}$, Shah AS, et al. Concomitant tricuspid valve surgery during implantation of continuous-flow left ventricular assist devices: A Society of Thoracic Surgeons database analysis. J Heart Lung Transplant 2014; 33: 609-617.

35. John R, Naka Y, Park SJ, Sai-Sudhakar C, Salerno C, Sundareswaran $\mathrm{KS}$, et al. Impact of concurrent surgical valve procedures in patients receiving continuous-flow devices. J Thorac Cardiovasc Surg 2014; 147: $581-589$.

36. Piacentino V III, Ganapathi AM, Stafford-Smith M, Hsieh MK, Patel $\mathrm{CB}$, Simeone AA, et al. Utility of concomitant tricuspid valve procedures for patients undergoing implantation of a continuous-flow left ventricular device. J Thorac Cardiovasc Surg 2012; 144: 1217 1221.

37. Piacentino V III, Troupes CD, Ganapathi AM, Blue LJ, Mackensen
GB, Swaminathan M, et al. Clinical impact of concomitant tricuspid valve procedures during left ventricular assist device implantation. Ann Thorac Surg 2011; 92: 1414-1418.

38. Groves DS, Blum FE, Huffmyer JL, Kennedy JL, Ahmad HB, Durieux ME, et al. Effects of early inhaled epoprostenol therapy on pulmonary artery pressure and blood loss during LVAD placement. J Cardiothorac Vasc Anesth 2014; 28: 652-660.

39. Potapov E, Meyer D, Swaminathan M, Ramsay M, el-Banayosy A, Diehl C, et al. Inhaled nitric oxide after left ventricular assist device implantation: A prospective, randomized, double-blind, multicenter, placebo-controlled trial. J Heart Lung Transplant 2011; 30: 870878.

40. Tector AJ. Transition from cardiopulmonary bypass to the HeartMate left ventricular assist device. Ann Thorac Surg 1998; 65: 643-646.

41. Scherer M, Sirat AS, Moritz A, Martens S. Extracorporeal membrane oxygenation as perioperative right ventricular support in patients with biventricular failure undergoing left ventricular assist device implantation. Eur J Cardiothorac Surg 2011; 39: 939-944.

42. Strueber M, Schmitto JD, Kutschka I, Haverich A. Placement of 2 implantable centrifugal pumps to serve as a total artificial heart after cardiectomy. J Thorac Cardiovasc Surg 2012; 143: 507-509.

43. Strueber M, Meyer AL, Malehsa D, Haverich A. Successful use of the HeartWare HVAD rotary blood pump for biventricular support. J Thorac Cardiovasc Surg 2010; 140: 936-937.

44. Krabatsch T, Potapov E, Stepanenko A, Schweiger M, Kukucka M, Huebler M, et al. Biventricular circulatory support with two miniaturized implantable assist devices. Circulation 2011; 124(Supp I): S179-S186.

45. McGee EC Jr, Ahmad U, Tamez D, Brown M, Voskoboynikov N, Malaisrie $\mathrm{C}$, et al. Biventricular continuous flow VADs demonstrate diurnal flow variation and lead to end-organ recovery. Ann Thorac Surg 2011; 92: e1-e3, doi:10.1016/j.athoracsur.2011.02.081.

46. Krabatsch T, Stepanenko A, Schweiger M, Kukucka M, Ewert P, Hetzer R, et al. Alternative technique for implantation of biventricular support with HeartWare implantable continuous flow pump. ASAIO J 2011; 57: 333-335.

\section{Supplementary Files}

Supplementary File 1

Appendix S1. INTERMACS definition of right heart failure

Please find supplementary file(s);

http://dx.doi.org/10.1253/circj.CJ-15-0093 